\title{
Artistic Expression of Urban Community Landscape Color
}

\author{
Hongxia Wang ${ }^{1}$, Huijing Wang ${ }^{1}$, Xiaomin $\mathrm{Hu}^{1}$ \\ ${ }^{1}$ College of Art and Design, Xingtai University, Xingtai, 054001, China
}

Keywords: urban community landscape color, function of color, component element, design principles

\begin{abstract}
Many people are fond of and appreciate dwelling environment with simple colors. At the same time, the research on urban community color and landscape is of important significance for the present urban community development and construction practices in China. Based on the statement on significance of urban community landscape color and functions of color, this paper analyzes component elements of community landscape color, specialty of design, factors influencing community landscape color and application of color in residential community landscape design, further analyzes design principles of urban community landscape color and then further discusses control and mode of urban community landscape color planning design.
\end{abstract}

\section{Introduction}

Based on the subject of color, Michael Lancaster, a British scholar, proposed the concept of

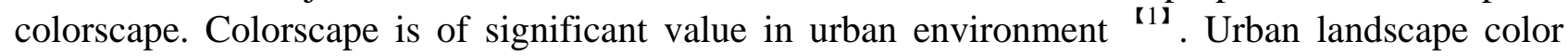
refers to the sum of all color elements in urban environment that can be visually perceived and reflected in an integrated and colonial appearance. Planning design of urban colorscape indicates visually pleasant urban landscape ${ }^{\mathbf{I} 2 \mathbf{I}}$ with regional characteristics and showing local culture that is formed by systematic and manipulative planning and design according to disciplinary theories related to urban environmental construction, physic-geographical environment, humanistic social environment and multiple limiting factors. Under current market-oriented economic conditions, urban community construction in China has already transformed from simple construction to emphasis on construction quality, environmental factors and landscape colors of communities. Therefore, the construction of urban communities with multiple functions and unique landscape colors becomes the most concerned issue of builders and residents.

\section{Concept and Significance of Urban Community Landscape Color}

Landscape color of urban community refers to the sum of all colors perceived out of all exposed objects in public space of a residential district. It is an abroad and integrated concept, which is divided into two categories: natural color and manual color ${ }^{\mathbf{r}}{ }^{3}$. Besides, landscape color of urban community includes the general term of visible colors of all plants, roads, waterscapes and others. Main landscaping elements of an urban community are plants as well as various landscapes, recreational facilities, boardwalks, parking spaces, public service facilities and so on. In order to provide people with convenient activity sites in individual urban community with large area and many houses, small plazas for collective activities of many people are usually constructed, supplemented with fountains, floor decorations, decorative ornaments or sports apparatuses. In such spatial region, colors of floors, water bodies and ornaments form main color of the urban community. However, colors of plants become match colors. Thus, people's activity space is formed by construction or design, having great effects on people's visual perception.

Color will deeply influence vital functions and psychology of a person while acting on his sensory organs. It is a visual element with the most expressive force and infectivity. To some extent, color even has stronger impact and attraction than the form of an object or a building. In urban community landscape, all component elements act on people's vision in a form of color. Therefore, color elements in urban community landscape are important means to improve function and role of dwelling environment. In urban community design, color and landscape should be contrastive and 
complementary. Influence, appetency and expression of landscape can be highlighted by unifying multiple colors into the overall color of urban community. Moreover, we should construct harmonious, peaceful and elegant urban community landscape color images with unique characteristics through design, planning and overall grasp, so as to remarkably improve functions and tastes of urban community.

\section{Functions of Color in Urban Community Landscape}

Color generates various functions through people's sensory stimulation, which not only can make people to have various complicated feelings (for instance, coldness, warmness, wideness, joy, sorrow, calmness, hotness, expansion, shrinkage, liveliness, depression, plainness, magnificence, association and symbolization) but also will affect people's emotions and psychologies and generate different aesthetic experiences. These feelings depend on emotional effects and characteristics of color itself. Therefore, we should lay emphasis on influencing function of color while designing and contrasting urban community landscape.

Psychosomatic Function of Color for People

Color refers to people's visual feelings towards visible lights. Color is also included in any design. Different people have different reactions for different colors. Through different colors, people can feel coldness, warmness, lightness, weight, softness, hardness, strength, weakness, brightness, darkness, tranquility, excitement, plainness and magnificence. These feelings depend on emotional effects and characteristics of color itself ${ }^{\mathbf{4} \mathbf{1}}$. Color refers to people's visual feelings towards visible lights. People's feelings and impressions towards color mainly include intuitive reactions and thought reactions. Intuitive reaction refers to people's first impression while seeing the color. This color phenomenon nearly generated by subconscious belongs to the scope of physiology. However, color feelings generated through careful observation and thinking for several times are products of thought, belonging to the scope of psychology. For instance, people will feel warm and hot when seeing red and yellow. However, people will feel cold and cool while seeing white, black and blue. These different physiological impressions generated due to different colors are mainly completed by people's physiological intuition and psychological association. Warm colors mainly include red and yellow; while cool colors mainly include blue and black. Neutral colors mainly include green and purple. However, there are subtle warmness and coldness differences among the three categories of color. Designers should attach great importance to subtle relations between color and psychology while designing landscape color.

Recognition Function of Color in Human Brain

People's recognition function for color is very important. At present, this recognition function has been widely applied into the environmental art design of urban community. However, how to let the Owner to improve his recognition function for urban community is an issue that a designer should be taken into first consideration. At present, landscape design of some large-scale urban communities lays particular stress on the use of natural color to strengthen the recognition function, for instance, yellow of Jacob's-rod, green of plants, cyan of hills and stones and multi-colors of flowers and plants. This recognition function relying on colors of landscapes and plants themselves is of great importance. Plant matching in landscape and ornaments should match with color design of the overall environmental planning, becoming a significant factor of landscape with distinctive features. Therefore, urban community designers can use identifiability of color itself for landscape design with distinctive differences. For instance, colors of sculptures, ornaments and waterscapes in landscape architecture can be used as important recognition means, not only displaying different functions and purposes but also deepening people's memory by means of colors with highlighted characters.

Aesthetic Function of Color for People

Harmonious and graceful color will arouse visual excitement and influence people's mental feelings. In terms of modeling, color can be used to adjust proportion, cover up flaws, highlight characteristics of the object itself and set off the object by contrast ${ }^{\mathbf{1 5 1}}$. Humans are not born with 
aesthetic perception. Also, such aesthetic perception is not obtained from imitation and learning. However, it is gradually formed and developed from social practices and productive labors. People's first impression on aesthetics of landscape is sensibility of color. The most principal function of color in landscape is aesthetic experience formed in human brain. Harmonious and perfect landscape color will motivate people's psychology of beauty and kindness, thereby arousing people's psychological and visual excitement and emotional resonance. Another function of color is to adjust the proportion of landscape modeling and play an important role in making good for deficiency and setting off artistic characteristics of modeling by contrast.

\section{Principal Factors Influencing Urban Community Landscape Color}

\section{Region and Climate}

Region and climate are principal factors influencing characteristics and development of urban community. Different regions and climates will form different urban community characteristics. For instance, thin shade landscape colors in coastal cities in the south and landscape colors of white walls and black tiles in Yangtze River Delta; climate differences between temperate zone and tropic zone and differences between plain urban community and mountainous or hilly urban community will form different community landscape colors. As northern regions are located in the subtemperate climatic zone and it is seriously affected by Siberian cold in winter, most communities are constructed in cool color tone, with most community landscapes supplemented with natural colors of hills, stones, pines and cypresses. Such communities are of typical community design mode with climatic characteristics in the north.

Historical Culture

Affected by historical and cultural factors, different cities will form different cultural characteristics. Likewise, urban communities will also form specific urban history and cultural features due to different humanistic colors and historical cultures. A piece of land with historical information available only and historical sites unavailable indicates that this land looses its historical content conceptions. Therefore, historical content conceptions should be selectively constructed, reappearing historical characteristics of this land to some extent and letting the community to produce poetic artistic conception, so as to avoid considerable similar regions and community landscapes lacking respective features.

\section{Community Scale}

Urban communities in different scales are different in development, building and mode of operation. Therefore, people's modes of feeling and experiencing community colorscape are different. In general, natural \& topographic conditions, size of a city and other factors will have an effect on the scale of residential district. In turn, the scale of residential district will also have specific effects on landscape design. In a specific community design, designers should carefully research geographic position of urban community, social status of residents, neighboring communities, production, development and multiple factors, so as to form the scale design mode complying with colorscape of this community.

Economic Factors

Community environment landscape planning is a long-term construction and development process. In this process, maintenance of landscape color and application cost are important factors that cannot be neglected. Such factors directly determine function exertion conditions of landscape as well as maintenance and protection effects of color. Thus, environmental design is made to not only realize deserved functions but also save economic costs and minimize economic operation costs.

\section{Design Principles of Urban Community Landscape Color}

The Principle of Overall Coordination

Colors of urban community landscapes (e.g. buildings, waterscapes, plants, trees, gardens and pavilions) are directly associated with integrality and harmony of community planning. Just as 
pointed by Schelling (a German philosopher) in the Philosophy of Art, "individual beauty is inexistent. Only integrity is beautiful ${ }^{\mathbf{6} 6 \mathbf{1}}$ ”. While planning and designing various landscapes, designers should start with overall layout of the community, specify a subject style, lay emphasis on changes in dominant tone, warm color tone and cold color tone of various landscapes, properly handling relations between manual colors, between manual colors and natural colors and between internal environment colors and external environment colors, use the artistic design mode of contrast colors to harmonize colors between different functional zones, and finally achieve the goal of harmony and unity.

The Principle of Natural Color

Natural color refers to essential colors of landscapes, buildings and materials. People have extremely strong capabilities to accept primary substances in the nature and prefer to natural colors, reflecting people's innermost requirements to long for returning to nature. People are fond of natural colors of trees, roads, landscapes and others alike. In terms of community landscape color, emphasis should be laid on maintenance and highlight of natural colors. Natural colors of landscapes can not only create a sense of returning to nature, but also give residents release space to relieve tension and mental stress.

The Principle of Humanization

Urban community is important living space of residents. Community landscape colors are directly correlated with living quality of people's daily activities, entertainment and physical training. Beautiful colors can adjust people's emotions and psychologies, letting people to have pleasant experiences. If colors are improperly matched, however, that will do harm to people's physical and psychological health. With respect to selection and matching of community landscape colors, relevant designers should master people's visual characteristics for colors, rationally use psychological effects of colors, or effectively improve tastes of the community and promote physical and psychological health of residents by beautifying color effects and modeling functions of landscapes.

The Principle of Local Features

In its long development history, each city will form respective humanistic and social features, with unique preferable color tone. To some extent, color reflects the maturity degree of culture. Urban community construction should take local features and climatic conditions into full consideration, so as to fully respect historical and cultural characteristics and embodying features, styles and cultural ethos of local cities and communities. For instance, northern people prefer landscapes with distinct colors; while southern people are fond of plain and light colors.

\section{Conclusions}

With continuous improvement to the level of civilization of human society, people have higher requirements for art and visual enjoyment. As an integral part of urban environment and carrier of urban history and culture, urban color begins to receive people's attention ${ }^{\text {[71 }}$. Urban community landscape color is an important component element of urban landscape, which deeply influences people's environmental feelings. To create vivid human settlement with distinct characters becomes people's general pursuit. The highest state of beautiful urban community landscape color is to present color artistic conception with charms of certain mental shackles and embody the landscape color function of grace, elegance and beautiful sceneries. With enhanced aesthetic experiences and improved aesthetic pursuits of people, urban community architectural color begins to present a trend of diversified development. Moreover, more emphasis begins to be laid onto overall harmony of color and effects on people's physiology, psychology and other aspects. Therefore, community design should comply with the principle of humanization, so as to provide people with more landscape colors with visual beauty, improve expression and influence of landscape color and promote health, harmony and unity between the community and people's life. 


\section{Acknowledgments}

This paper is the research achievement of A Research on Urban Community Landscape Colors in the 2014 humanistic and social science research project held by institutions of higher learning in Hebei Province.

\section{References:}

[1] Zhang Xiaokai and Shang Jinkai, Research on Color Characteristics of Urban Landscape - with Tianjin as an Example [J], Art and Design (Theory) 2010 (5): 130-132;

[2] Yang Ning, Urban Color Art and Urban Landscape Characteristics [J], Urban Construction Theory Research, 2013 (47): 212-213;

[3] Ma Weihua, Peng Zhonghua and Li Lin, Landscape Colors of Residential Areas [J], Shanxi Architecture, 2007, 33 (8): 49-51;

[4] Li Xing, Design on Colors of Urban Landscape [J], Tianjin University, 2012 (7):1-78;

[5] Wang He, Landscape Design of Modern Urban Community [J], Beauty \& Times, 2010 (6): 100-102;

[6] Jiang Fang and Huang Weixiu, A Discussion on Landscape Design of Urban Colors - with Wuhan Urban Colorscape as an Example [J], Journal of Hubei University of Education, 2009, 26 (8):121-122+139;

[7] Luo Hong’an, Design Principles and Measures for Landscape Planning of Residential Districts [J], Journal of Anhui Agricultural Sciences, 2011 (13): 57-58+217. 